\title{
IGF-1 and cardiometabolic diseases: a Mendelian randomisation study
}

\author{
Susanna C. Larsson $^{1,2}$ (D) Karl Michaëlsson ${ }^{1}$ (D) $\cdot$ Stephen Burgess $^{3,4}$ (D)
}

Received: 22 February 2020 / Accepted: 21 April 2020 / Published online: 16 June 2020

(C) The Author(s) 2020

\begin{abstract}
Aims/hypothesis Abnormal serum IGF-1 levels are associated with an increased risk of type 2 diabetes and cardiovascular disease. However, the causal role of IGF-1 levels within the normal range in cardiometabolic disease remains unclear. We employed Mendelian randomisation to explore the associations between genetically predicted serum IGF-1 levels and cardiometabolic diseases.

Methods Serum IGF-1 levels were predicted using 416 SNPs associated with IGF-1 levels among 358,072 individuals in UK Biobank. Genetic association estimates for the outcomes were obtained from consortia of type 2 diabetes (74,124 cases, 824,006 controls), coronary artery disease (60,801 cases, 123,504 controls), heart failure (47,309 cases, 930,014 controls), atrial fibrillation (65,446 cases, 522,744 controls), and ischaemic stroke (60,341 cases, 454,450 controls).

Results Genetic predisposition to elevated serum IGF-1 levels was associated with higher risk of type 2 diabetes and coronary artery disease. The OR (95\% CI) per SD increment in IGF-1 level was $1.14(1.05,1.24)$ for type 2 diabetes and 1.09 (1.02, 1.16$)$ for coronary artery disease. The association between IGF-1 and coronary artery disease was attenuated after adjustment for type 2 diabetes (OR 1.06 [95\% CI 1.00, 1.13]), suggesting that the association may be partly mediated via type 2 diabetes. There was limited evidence of associations between IGF-1 levels and heart failure, atrial fibrillation and ischaemic stroke.

Conclusions/interpretation This study found evidence that increased IGF-1 levels may be causally associated with higher risk of type 2 diabetes.
\end{abstract}

Keywords IGF-1 $\cdot$ Insulin-like growth factor $\cdot$ Mendelian randomisation $\cdot$ Type 2 diabetes

\section{Abbreviations}

AFGen Atrial Fibrillation Consortium

DIAGRAM Diabetes Genetics Replication and Metaanalysis

Electronic supplementary material The online version of this article (https://doi.org/10.1007/s00125-020-05190-9) contains peer-reviewed but unedited supplementary material, which is available to authorised users.

Susanna C. Larsson

susanna.larsson@surgsci.uu.se

1 Department of Surgical Sciences, Uppsala University, Epihubben, 75185 Uppsala, Sweden

2 Unit of Cardiovascular and Nutritional Epidemiology, Institute of Environmental Medicine, Karolinska Institutet, Stockholm, Sweden

3 Department of Public Health and Primary Care, University of Cambridge, Cambridge, UK

4 MRC Biostatistics Unit, University of Cambridge, Cambridge, UK

$\begin{array}{ll}\text { GWAS } & \text { Genome-wide association study } \\ \text { IVW } & \text { Inverse-variance weighted } \\ \text { MR } & \text { Mendelian randomisation } \\ \text { PRESSO } & \text { Pleiotropy Residual Sum and Outlier }\end{array}$

\section{Introduction}

IGF-1 is a polypeptide hormone that is structurally similar to proinsulin. IGF-1 is synthesised primarily in the liver upon stimulation by growth hormone and is a key mediator of growth hormone-stimulated growth and other anabolic activities in many cells and tissues [1]. Both pathological excess, as in acromegaly, and deficiency of IGF-1 are associated with glucose intolerance, insulin resistance and increased risk of type 2 diabetes and cardiovascular morbidity and mortality $[1,2]$. However, the effects of high serum IGF-1 levels within the normal range on cardiometabolic diseases remains 


\section{Research in context}

\section{What is already known about this subject?}

- Both pathological excess, as in acromegaly, and deficiency of IGF-1 are associated with increased risk of type 2 diabetes and cardiovascular disease

- Results of observational studies of circulating IGF-1 levels within the normal range in relation to cardiometabolic diseases are inconclusive

\section{What is the key question?}

- Are high serum IGF-1 levels within the normal range causally associated with type 2 diabetes and cardiovascular disease?

What are the new findings?

- Genetically higher serum IGF-1 levels were associated with increased risk of type 2 diabetes and coronary artery disease

- The association between IGF-1 and coronary artery disease appeared to be mediated, at least partly, via type 2 diabetes

- There was limited evidence of associations of IGF-1 with heart failure, atrial fibrillation and ischaemic stroke

How might this impact on clinical practice in the foreseeable future?

- IGF-1 levels can be modified by milk and protein intake and, thus, a reduced protein intake may lower type 2 diabetes risk

unclear. Several observational studies [3-15], although not all [16-19], have reported the association of either low or high circulating total IGF-1 levels with type 2 diabetes and different cardiovascular diseases. Nevertheless, because underlying disease may influence IGF-1 levels, and observational studies are vulnerable to confounding, causality cannot be inferred based on available data.

Mendelian randomisation (MR) is a method to address causality in observational studies using one or multiple genetic variants affecting the risk factor as a genetic instrument for the effect of the risk factor on disease. Here, we used the MR design to investigate the associations of long-term increased IGF-1 levels with type 2 diabetes and major cardiovascular diseases. In secondary analyses, we explored the associations of genetically predicted IGF-1 levels with components of the metabolic syndrome. Given the important role of IGF-1 in growth, we also assessed the association of genetically predicted IGF-1 levels with height, as a positive control.

\section{Methods}

Genetic instrument for IGF-1 Instrumental variables for serum IGF-1 levels were selected from a genome-wide association study (GWAS) of 358,072 European-descent individuals in UK Biobank [20]. Among the genome-wide significant $\left(p<5 \times 10^{-8}\right)$ SNPs identified in that GWAS, we selected 416 SNPs after exclusion of correlated SNPs based on a linkage disequilibrium threshold of $R^{2}<0.01$. The SNPs were estimated to explain $9.4 \%$ of the variance in IGF-1 levels, and the $F$ statistic was 80.9. In UK Biobank the mean (range) age of participants is 56.5 (37-73) years, the mean (SD) IGF-1 concentration is $21.4(5.7) \mathrm{nmol} / \mathrm{l}$ and the IGF-1 concentration in the first and ninth decile is $14.2 \mathrm{nmol} / \mathrm{l}$ and $28.4 \mathrm{nmol} / \mathrm{l}$, respectively.

Outcome data sources Summary-level data for the genetic associations with the outcomes were obtained from metaanalyses of GWASs [21-33] or UK Biobank for BP. We used the largest publicly available GWAS dataset for each exposure, except for the glycaemic traits where the largest GWAS dataset [34] only included 29 of the 416 SNPs for IGF-1. Information on the data sources is provided in Table 1. In brief, for the cardiometabolic diseases, we used data from the Atrial Fibrillation Consortium (AFGen) [26, 27], the Coronary Artery Disease Genome-wide Replication and Meta-analysis plus The Coronary Artery Disease Genetics consortium [24], the Diabetes Genetics Replication and Meta-analysis (DIAGRAM) consortium [21, 22], the Heart Failure Molecular Epidemiology for Therapeutic Targets (HERMES) consortium [28] and the MEGASTROKE consortium [25]. For atrial fibrillation and type 2 diabetes, we additionally used data from the FinnGen consortium [23], which had no sample overlap with the AFGen and DIAGRAM consortia. However, some studies in the FinnGen consortium were part of the other consortia and 
Table 1 Data sources for the outcomes

\begin{tabular}{|c|c|c|c|c|c|}
\hline Outcome & $\begin{array}{l}\text { No. of } \\
\text { cases }\end{array}$ & $\begin{array}{l}\text { No. of } \\
\text { controls }\end{array}$ & Population & $\begin{array}{l}\text { No. of SNPs } \\
\text { used for } \\
\text { IGF-1 }\end{array}$ & Consortium \\
\hline
\end{tabular}

\begin{tabular}{|c|c|c|c|c|c|}
\hline \multicolumn{6}{|l|}{ Cardiometabolic disease } \\
\hline Type 2 diabetes & 74,124 & 824,006 & European & 416 & DIAGRAM [21] \\
\hline Type 2 diabetes ${ }^{\mathrm{a}}$ & 26,676 & 132,532 & European & 416 & DIAGRAM [22] \\
\hline Type 2 diabetes ${ }^{\mathrm{a}}$ & 11,006 & 82,655 & European & 393 & FinnGen [23] \\
\hline $\begin{array}{l}\text { Coronary artery } \\
\text { disease }\end{array}$ & 60,801 & 123,504 & Mixed & 408 & $\begin{array}{l}\text { CARDIoGRAMplusC4D } \\
\text { [24] }\end{array}$ \\
\hline Atrial fibrillation & 65,446 & 522,744 & Mixed & 416 & AFGen (2018 dataset) [26] \\
\hline Atrial fibrillation $^{\mathrm{a}}$ & 17,931 & 115,142 & Mixed & 416 & AFGen (2017 dataset) [27] \\
\hline Atrial fibrillation $^{\mathrm{a}}$ & 7244 & 56,378 & European & 393 & FinnGen [23] \\
\hline Heart failure & 47,309 & 930,014 & European & 413 & HERMES [28] \\
\hline Ischaemic stroke & 60,341 & 454,450 & Mixed & 398 & MEGASTROKE [25] \\
\hline \multicolumn{6}{|l|}{ Glycaemic traits ${ }^{\mathrm{b}}$} \\
\hline Fasting glucose & NA & 46,186 & European & 297 & MAGIC [29] \\
\hline Fasting insulin & NA & 38,238 & European & 298 & MAGIC [29] \\
\hline HOMA-IR & NA & 46,187 & European & 297 & MAGIC [29] \\
\hline \multicolumn{6}{|l|}{ Serum lipids ${ }^{\mathrm{b}}$} \\
\hline HDL-cholesterol & NA & 187,167 & Mixed & 296 & GLGC [30] \\
\hline LDL-cholesterol & NA & 173,083 & Mixed & 296 & GLGC [30] \\
\hline Total cholesterol & NA & 187,365 & Mixed & 296 & GLGC [30] \\
\hline Triacylglycerols & NA & 177,861 & Mixed & 296 & GLGC [30] \\
\hline \multicolumn{6}{|l|}{$\mathrm{BP}^{\mathrm{b}}$} \\
\hline Systolic BP & NA & 317,754 & European & 414 & UK Biobank (Neale Lab) \\
\hline Diastolic BP & NA & 317,756 & European & 414 & UK Biobank (Neale Lab) \\
\hline \multicolumn{6}{|l|}{ Body composition $^{\mathrm{b}}$} \\
\hline BMI & NA & 339,224 & Mixed & 297 & GIANT [31] \\
\hline Waist circumference & NA & 224,459 & European & 297 & GIANT [32] \\
\hline WHR & NA & 224,459 & European & 297 & GIANT [32] \\
\hline Height & NA & 253,288 & European & 296 & GIANT [33] \\
\hline
\end{tabular}

${ }^{\mathrm{a}}$ This data source did not have participant overlap with the data source for the exposure (IGF-1 levels) and was used as a supplementary analysis

${ }^{\mathrm{b}}$ Summary association estimates for these outcomes were obtained through the MR-Base platform (database version 0.2.0, 17 December 2017) [40]

CARDIoGRAMplusC4D, Coronary Artery Disease Genome-wide Replication and Meta-analysis plus The Coronary Artery Disease Genetics; GIANT, Genetic Investigation of Anthropometric Traits; GLGC, Global Lipids Genetics Consortium; HERMES, Heart Failure Molecular Epidemiology for Therapeutic Targets; MAGIC, Meta-Analyses of Glucose and Insulin-related traits Consortium therefore not used for other outcomes. For SNPs that were unavailable in the outcome dataset, proxy SNPs in linkage disequilibrium $\left(R^{2} \geq 0.8\right)$ with the IGF-1-associated SNPs were used when available. Most GWASs adjusted for sex and genetic principal components. Summary statistics for the SNPs related to IGF-1 levels and the corresponding statistics for the cardiometabolic diseases are presented in electronic supplementary material (ESM) Tables 1-5. All studies included in the GWASs had been approved by an ethical review committee, and participants provided informed consent. This MR study was approved by the Swedish Ethical Review Authority.
Statistical analysis The MR estimates were obtained using the inverse-variance weighted (IVW) method under a multiplicative random-effects model. The $I^{2}$ statistic was used to assess heterogeneity between the estimates obtained from individual SNPs. Sensitivity analyses using the weighted median [35], MR Pleiotropy Residual Sum and Outlier (PRESSO) [36] and MR-Egger [35] methods were conducted. The weighted median method provides a valid estimate if at least $50 \%$ of the weight originates from non-pleiotropic SNPs. The MRPRESSO method and MR-Egger method can adjust for potential outliers and directional pleiotropy, respectively. To address possible pleiotropy with other members of the IGF 
axis, we conducted a sensitivity analysis excluding three SNPs in the $I G F B P 3$ or $I G F 2$ gene regions. Multivariable MR analysis was used to evaluate the direct effect of IGF-1 levels on cardiometabolic diseases not mediated by fasting insulin levels, insulin resistance or height. This analysis was carried out to assess whether any association between IGF-1 and the cardiometabolic diseases could be mediated by those factors. Multivariable MR analysis [37] was also used to estimate the direct effect of IGF-1 levels on coronary artery disease not mediated via type 2 diabetes.

Due to partial sample overlap in the data source for IGF-1 and the largest GWASs for type 2 diabetes [21] and atrial fibrillation [26], we performed a supplementary analysis using data from previous smaller GWASs for these outcomes [22, 27] and the FinnGen consortium [23] that did not include UK Biobank. The heart failure dataset also included UK Biobank participants but no other large and publicly available GWAS dataset without UK Biobank was available, and the FINRISK study from the FinnGen consortium were included in the heart failure GWAS. There was no sample overlap for the data source for IGF-1 and the datasets for coronary artery disease and ischaemic stroke.

All presented results are expressed per SD increase in IGF1 levels (equivalent to about $5.7 \mathrm{nmol} / \mathrm{l}$ in UK Biobank). The statistical analyses were performed using the mrrobust package in Stata [38], the MendelianRandomization package in $\mathrm{R}$ [39] and the MR-Base platform [40].

\section{Results}

Cardiometabolic diseases Genetically predicted IGF-1 levels were positively associated with type 2 diabetes and coronary artery disease in the primary (IVW) analysis (Fig. 1). The OR (95\% CI) per SD increase in genetically predicted IGF-1 levels was $1.14(1.05,1.24)$ for type 2 diabetes and 1.09 $(1.02,1.16)$ for coronary artery disease. The association with type 2 diabetes was confirmed when using data from a smaller dataset from the DIAGRAM consortium that did not include UK Biobank (OR 1.13 [95\% CI 1.03, 1.25]) and in the FinnGen consortium (OR 1.20 [95\% CI 1.08, 1.34]). Furthermore, the association between genetically predicted IGF-1 levels and type 2 diabetes was consistent in sensitivity analyses based on the weighted median and MR-PRESSO methods (Fig. 1). The OR estimate for type 2 diabetes did not change after exclusion of three SNPs in the IGFBP3 or $I G F 2$ gene regions. The MR-Egger analysis showed no indication of directional pleiotropy ( $p$ for intercept $=0.464)$. The association between IGF-1 and coronary artery disease persisted in the MR-PRESSO analysis but not in the weighted median and MR-Egger analyses; however, the precision was low in the MR-Egger analysis and there was no evidence of directional pleiotropy $(p$ for intercept $=0.116)($ Fig. 1$)$.
Exclusion of the three SNPs in the IGFBP3 or IGF2 gene regions did not essentially alter the results for coronary artery disease (OR 1.10 [95\% CI 1.03, 1.17]).

There was no strong evidence for associations between IGF-1 levels and heart failure (OR 1.01 [95\% CI 0.97, 1.06]), atrial fibrillation (OR 1.03 [95\% CI 0.97, 1.08]) or ischaemic stroke (OR 1.03 [95\% CI 0.98, 1.08]) (Fig. 1). For atrial fibrillation, the OR was $1.05(95 \%$ CI $0.98,1.13)$ when using a smaller GWAS dataset from the AFGen that did not include UK Biobank, and the OR was 0.92 (95\% CI 0.82, $1.04)$ in the FinnGen consortium. For subtypes of ischaemic stroke, the ORs $(95 \%$ CIs) were $0.93(0.83,1.04)$ for large artery stroke, $1.10(0.99,1.06)$ for small vessel stroke and 1.06 $(0.96,1.16)$ for cardioembolic stroke.

Components of the metabolic syndrome and height There was strong or suggestive evidence that genetically higher IGF-1 levels were associated with higher fasting glucose $\left(p=6.96 \times 10^{-3}\right)$ and insulin $\left(p=6.31 \times 10^{-5}\right)$ levels, increased insulin resistance $\left(p=6.63 \times 10^{-5}\right)$, higher diastolic $\mathrm{BP}(p=0.016)$, lower total cholesterol $(p=0.021)$ and triacylglycerol $\left(p=1.31 \times 10^{-3}\right)$ levels and higher height $(p=6.46 \times$ $10^{-4}$ ) in the primary analysis (Fig. 2). The most consistent associations across sensitivity analyses were with fasting insulin levels, insulin resistance and height (Fig. 2).

Direct effects of IGF-1 The associations of genetically predicted IGF-1 levels with type 2 diabetes and coronary artery disease were similar after adjustment for insulin levels (ESM Table 6) or insulin resistance (ESM Table 7), whereas adjustment for height resulted in somewhat stronger associations (ESM Table 8). The lack of associations with the other outcomes persisted after adjustment for insulin levels, insulin resistance and height (ESM Tables 6-8). The association between genetically predicted IGF-1 levels and coronary artery disease was attenuated after adjustment for type 2 diabetes (OR 1.06 [95\% CI 1.00, 1.13], $p=0.063$ ).

\section{Discussion}

This MR study showed that genetically higher IGF-1 levels were associated with increased risk of type 2 diabetes and coronary artery disease, though results for coronary artery disease were not consistent across all sensitivity analyses. Genetically higher IGF1 levels were additionally associated with some components of the metabolic syndrome, the most robust association being with fasting insulin and insulin resistance.

Our findings are in line with the results of two nested casecontrol studies, which demonstrated that high IGF-1 levels were associated with a statistically significant [10] or a suggestive [15] increased risk of type 2 diabetes. Another nested case-control study found that high levels of free IGF-1 were associated with 


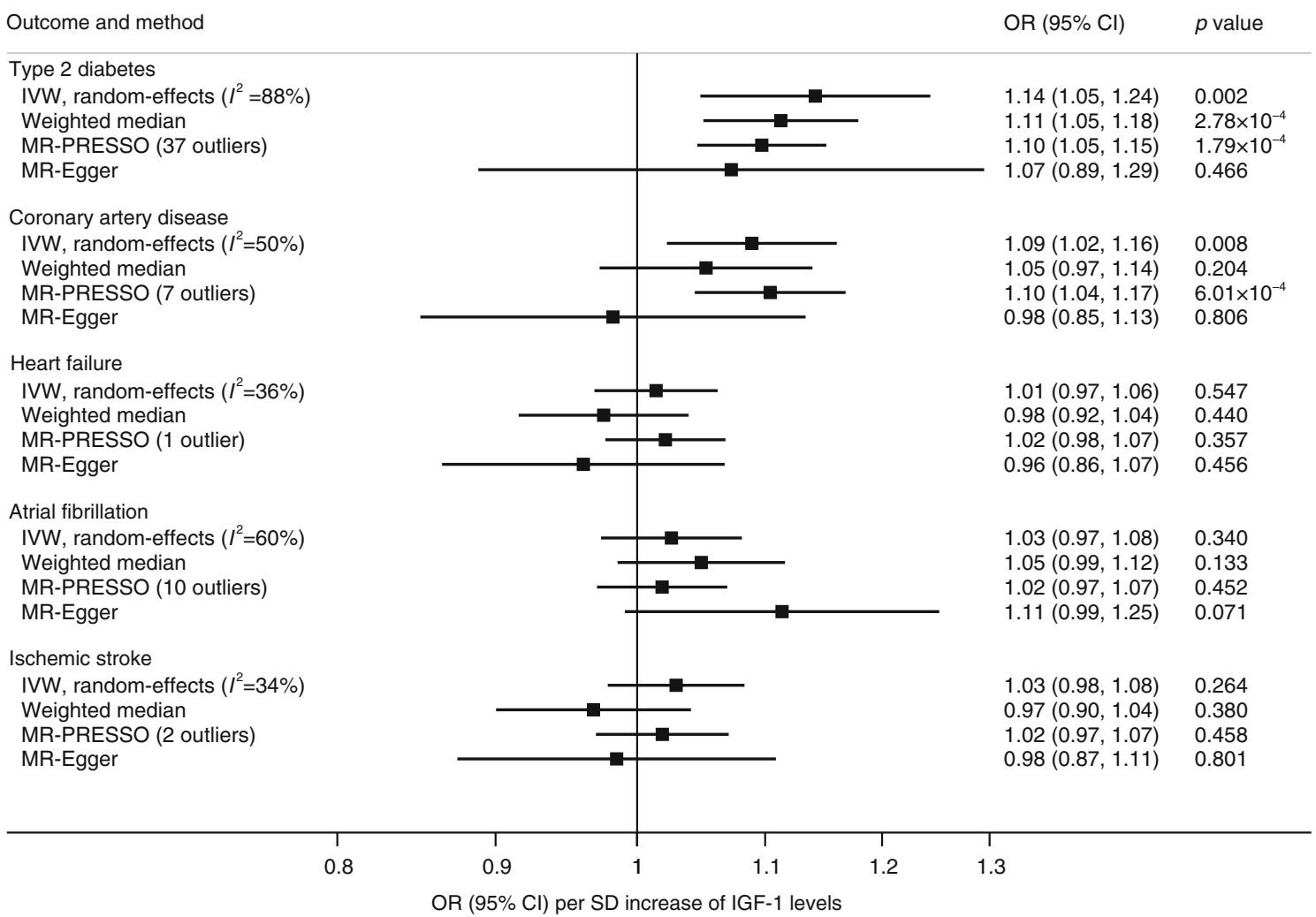

Fig. 1 Associations between genetically predicted IGF-1 levels and type 2 diabetes and cardiovascular diseases

higher risk of type 2 diabetes in individuals with insulin levels above the median, but with lower risk in individuals with insulin levels below the median [41]. However, a null association between IGF-1 levels and type 2 diabetes has also been reported $[9,18]$ and a cohort study of 615 participants showed that the 51 participants who developed impaired glucose tolerance or type 2 diabetes during a follow-up period of 4.5 years had lower IGF-1 levels compared with those who did not develop impaired glucose tolerance [5]. The reason for these conflicting results is unclear. Nevertheless, the relatively small sample sizes (ranging from around 50 to 800 cases) in previous studies and potential reverse causality, whereby the disease process caused changes in IGF-1 levels several years before the clinical diagnosis of type 2 diabetes, may have resulted in null or spurious findings.

Our findings for IGF-1 levels and cardiovascular disease corroborate those of some but not all observational studies. In a cohort study of 2901 Swedish men (including 589 incident cardiovascular events), both high ( $>80$ th percentile) and low $(<20$ th percentile) IGF-1 levels were associated with increased risk of any cardiovascular event, and high but not low IGF-1 levels were associated with a statistically significant higher risk of coronary artery disease [12]. Similarly, in a nested casecontrol study of US women (245 myocardial infarction cases and 490 matched controls), the multivariable-adjusted RR (95\% CI) of myocardial infarction was $2.09(1.17,3.72)$ and $1.46(0.79,2.72)$ for the third and fourth highest quartile of IGF-1 levels, respectively, when compared with the bottom quartile [8]. IGF-1 levels were also positively associated with coronary artery disease in a cross-sectional study of 6773 German adults [9]. In contrast, some other cross-sectional and nested case-control studies (including 57-374 cases) reported that elevated IGF-1 levels were associated with lower prevalence or incidence of coronary artery disease $[3,4,11]$, heart failure [6], atrial fibrillation [13] and ischaemic stroke [7, 14]. Other crosssectional and nested case-control studies showed a null association between IGF-1 levels and coronary artery disease (1671013 cases) $[16,17,19]$ and cerebrovascular events (273 cases) [12]. The inconsistent results might reflect small sample sizes in most previous studies, reverse causality or residual confounding.

The main strength of this study is the MR design, which reduced confounding and reverse causation bias. Another strength is the large sample sizes for both the data source (UK Biobank) used to derive the genetic association with IGF-1 levels and for the data sources used for genetic associations with the outcomes. This, along with the strong genetic instrument for serum IGF-1 levels, resulted in high precision of the results in the primary analyses. The estimates obtained from the MREgger analysis were imprecise and should be interpreted with caution. A further strength is that a large number of SNPs was available as instrumental variables for IGF-1 levels. We could therefore conduct several sensitivity analyses to evaluate pleiotropy. A limitation of this study is that the genetic instrument was for total IGF-1 levels rather than the free and bioavailable IGF-1 fraction, which may be more strongly associated with type 2 


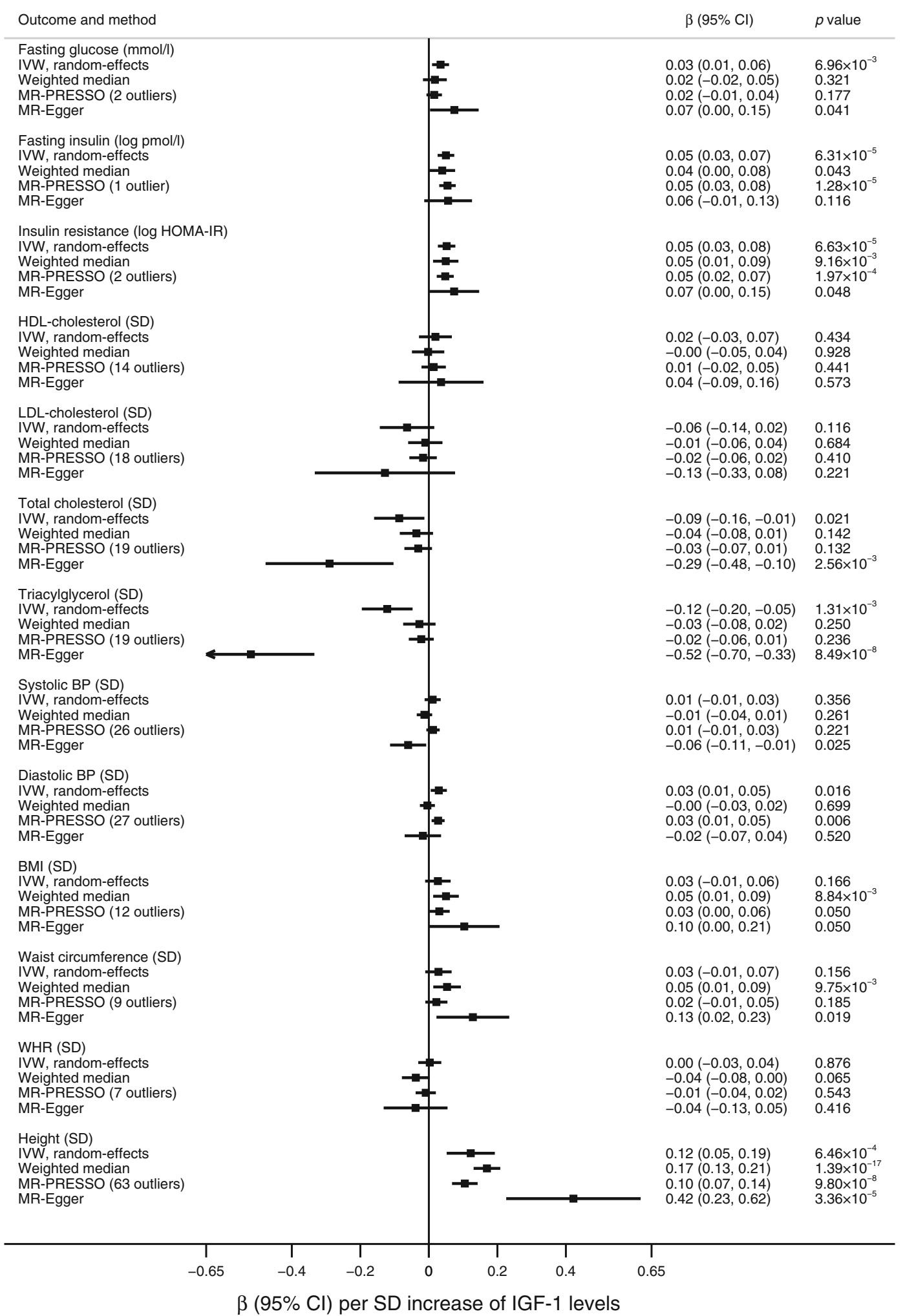

Fig. 2 Associations between genetically predicted IGF-1 levels and components of the metabolic syndrome and height. Log indicates natural logarithmic transformed levels $\left(\log _{e}\right)$

diabetes and cardiovascular diseases. Another shortcoming is that we could not investigate whether there is a U- or J-shaped relationship between IGF-1 levels and cardiometabolic diseases and insulin resistance, as suggested by a few observational studies $[12,42]$. However, confounding by height might have resulted in non-linear associations in those studies. 
In this study, we found that the association between genetically predicted serum IGF-1 levels and type 2 diabetes was partially attenuated after adjustment for fasting insulin levels or insulin resistance through multivariable MR analysis. This suggests that elevated IGF-1 levels may increase the risk of type 2 diabetes in part through insulin resistance. Further research is needed to understand other possible mechanisms underlying the association between IGF-1 and type 2 diabetes. The association between IGF-1 levels and coronary artery disease appeared to be mediated, at least partly, via type 2 diabetes.

Evidence indicates that IGF-1 levels may be modified by milk and protein intake [43-47]. A meta-analysis of eight randomised controlled trials showed a statistically significant $13.8 \mathrm{ng} / \mathrm{ml}$ (equivalent to about $1.8 \mathrm{nmol} / \mathrm{l}$ ) difference in IGF-1 levels when comparing the milk intervention group with the control group [44]. Furthermore, several randomised controlled trials have demonstrated that increased dietary protein intake or whey protein (one of the two proteins in milk) supplementation increase circulating IGF-1 levels [45-47]. Intake of protein, particularly from animal sources, has been found to be positively associated with type 2 diabetes risk in observational studies [48]. Additionally, evidence from experimental, observational and MR studies indicates that high circulating levels of branchedchain amino acids, found in high levels in for example whey protein, increase the risk of type 2 diabetes [49].

Conclusions This MR study found evidence of a causal association between increased IGF-1 levels within the normal range and higher risk of type 2 diabetes. This finding may have public health and clinical implications as IGF-1 levels may be modified by milk and protein intake [43-47].

\begin{abstract}
Acknowledgements The authors acknowledge the participants and investigators of all consortia that contributed summary statistics data, including the AFGen, Coronary Artery Disease Genome-wide Replication and Meta-analysis plus The Coronary Artery Disease Genetics consortium, DIAGRAM consortium, FinnGen consortium, Genetic Investigation of Anthropometric Traits, Global Lipids Genetics Consortium, HERMES consortium, Meta-Analyses of Glucose and Insulin-related traits Consortium and the MEGASTROKE consortium. The list of investigators of the MEGASTROKE consortium is available at http://megastroke.org/ authors.html, and the funding sources of the MEGASTROKE project is specified at megastroke.org/acknowledgements.html.
\end{abstract}

Data availability All data analysed in this study are based on publicly available summary statistics data provided by genetic consortia.

Funding Open access funding provided by Uppsala University. This study was supported by the Swedish Research Council (Vetenskapsrådet grant no. 2019-00977), the Swedish Research Council for Health, Working Life and Welfare (Forte grant no. 2018-00123) and the Swedish Heart-Lung Foundation (Hjärt-Lungfonden grant no. 20190247). Stephen Burgess is supported by Sir Henry Dale Fellowship jointly funded by the Wellcome Trust and the Royal Society (grant no. 204623/Z/16/Z).

Authors' relationships and activities The authors declare that there are no relationships or activities that might bias, or be perceived to bias, their work.
Contribution statement SCL designed the study, performed the statistical analyses, wrote the manuscript and created tables and figures. $\mathrm{KM}$ and $\mathrm{SB}$ interpreted the data and edited the manuscript. All authors have given final approval of the version to be published. SCL is the guarantor of this study and, as such, had full access to all of the data in the study and takes responsibility for the integrity of the data and the accuracy of the data analysis.

Open Access This article is licensed under a Creative Commons Attribution 4.0 International License, which permits use, sharing, adaptation, distribution and reproduction in any medium or format, as long as you give appropriate credit to the original author(s) and the source, provide a link to the Creative Commons licence, and indicate if changes were made. The images or other third party material in this article are included in the article's Creative Commons licence, unless indicated otherwise in a credit line to the material. If material is not included in the article's Creative Commons licence and your intended use is not permitted by statutory regulation or exceeds the permitted use, you will need to obtain permission directly from the copyright holder. To view a copy of this licence, visit http://creativecommons.org/licenses/by/4.0/.

\section{References}

1. Hannon AM, Thompson CJ, Sherlock M (2017) Diabetes in patients with acromegaly. Curr Diab Rep 17:8

2. Lombardi G, Di Somma C, Grasso LF, Savanelli MC, Colao A, Pivonello R (2012) The cardiovascular system in growth hormone excess and growth hormone deficiency. J Endocrinol Investig 35: $1021-1029$

3. Janssen JA, Stolk RP, Pols HA, Grobbee DE, Lamberts SW (1998) Serum total IGF-I, free IGF-I, and IGFB-1 levels in an elderly population: relation to cardiovascular risk factors and disease. Arterioscler Thromb Vasc Biol 18:277-282

4. Juul A, Scheike T, Davidsen M, Gyllenborg J, Jorgensen T (2002) Low serum insulin-like growth factor I is associated with increased risk of ischemic heart disease: a population-based case-control study. Circulation 106:939-944

5. Sandhu MS, Heald AH, Gibson JM, Cruickshank JK, Dunger DB, Wareham NJ (2002) Circulating concentrations of insulin-like growth factor-I and development of glucose intolerance: a prospective observational study. Lancet 359:1740-1745

6. Vasan RS, Sullivan LM, D Agostino RB et al (2003) Serum insulin-like growth factor I and risk for heart failure in elderly individuals without a previous myocardial infarction: the Framingham Heart Study. Ann Intern Med 139:642-648

7. Johnsen SP, Hundborg HH, Sorensen HT et al (2005) Insulin-like growth factor (IGF) I, -II, and IGF binding protein-3 and risk of ischemic stroke. J Clin Endocrinol Metab 90:5937-5941

8. Page JH, Ma J, Pollak M, Manson JE, Hankinson SE (2008) Plasma insulinlike growth factor 1 and binding-protein 3 and risk of myocardial infarction in women: a prospective study. Clin Chem 54:1682-1688

9. Schneider HJ, Klotsche J, Saller B et al (2008) Associations of agedependent IGF-I SDS with cardiovascular diseases and risk conditions: cross-sectional study in 6773 primary care patients. Eur J Endocrinol 158:153-161

10. Lewitt MS, Hilding A, Brismar K, Efendic S, Ostenson CG, Hall K (2010) IGF-binding protein 1 and abdominal obesity in the development of type 2 diabetes in women. Eur J Endocrinol 163:233-242

11. Ruidavets JB, Luc G, Machez E et al (2011) Effects of insulin-like growth factor 1 in preventing acute coronary syndromes: the PRIME study. Atherosclerosis 218:464-469 
12. Carlzon D, Svensson J, Petzold M et al (2014) Both low and high serum IGF-1 levels associate with increased risk of cardiovascular events in elderly men. J Clin Endocrinol Metab 99:E2308-E2316

13. Duron E, Vidal JS, Funalot B et al (2014) Insulin-like growth factor I, insulin-like growth factor binding protein 3, and atrial fibrillation in the elderly. J Gerontol A Biol Sci Med Sci 69:1025-1032

14. Saber H, Himali JJ, Beiser AS et al (2017) Serum insulin-like growth factor 1 and the risk of ischemic stroke: the Framingham Study. Stroke 48:1760-1765

15. Simila ME, Kontto JP, Virtamo J et al (2019) Insulin-like growth factor I, binding proteins -1 and -3 , risk of type 2 diabetes and macronutrient intakes in men. Br J Nutr 121:938-944

16. Lawlor DA, Ebrahim S, Smith GD, Cherry L, Watt P, Sattar N (2008) The association of insulin-like-growth factor 1 (IGF-1) with incident coronary heart disease in women: findings from the prospective British Women s Heart and Health Study. Atherosclerosis 201:198-204

17. Ricketts SL, Rensing KL, Holly JM et al (2011) Prospective study of insulin-like growth factor-I, insulin-like growth factor-binding protein 3, genetic variants in the IGF1 and IGFBP3 genes and risk of coronary artery disease. Int J Mol Epidemiol Genet 2:261-285

18. Drogan D, Schulze MB, Boeing H, Pischon T (2016) Insulin-like growth factor 1 and insulin-like growth factor-binding protein 3 in relation to the risk of type 2 diabetes mellitus: results from the EPIC-Potsdam Study. Am J Epidemiol 183:553-560

19. Kaplan RC, McGinn AP, Pollak MN et al (2007) Association of total insulin-like growth factor-I, insulin-like growth factor binding protein-1 (IGFBP-1), and IGFBP-3 levels with incident coronary events and ischemic stroke. J Clin Endocrinol Metab 92:1319-1325

20. Sinnott-Armstrong N, Tanigawa Y, Amar D, et al (2019) Genetics of 38 blood and urine biomarkers in the UK Biobank. Available from https://www.biorxiv.org/content/10.1101/660506v1. Accessed 29 March 2020

21. Mahajan A, Taliun D, Thurner M et al (2018) Fine-mapping type 2 diabetes loci to single-variant resolution using high-density imputation and islet-specific epigenome maps. Nat Genet 50:1505-1513

22. Scott RA, Scott LJ, Magi R et al (2017) An expanded genome-wide association study of type 2 diabetes in Europeans. Diabetes 66:28882902

23. FinnGen consortium (2020). FinnGen documentation of R2 release, 2020. Available from https://finngen.gitbook.io/documentation/. Accessed 29 March 2020

24. Nikpay M, Goel A, Won HH et al (2015) A comprehensive 1,000 genomes-based genome-wide association meta-analysis of coronary artery disease. Nat Genet 47:1121-1130

25. Malik R, Chauhan G, Traylor M et al (2018) Multiancestry genomewide association study of 520,000 subjects identifies 32 loci associated with stroke and stroke subtypes. Nat Genet 50:524-537

26. Roselli C, Chaffin MD, Weng LC et al (2018) Multi-ethnic genome-wide association study for atrial fibrillation. Nat Genet 50:1225-1233

27. Christophersen IE, Rienstra M, Roselli C et al (2017) Large-scale analyses of common and rare variants identify 12 new loci associated with atrial fibrillation. Nat Genet 49:946-952

28. Shah S, Henry A, Roselli C et al (2020) Genome-wide association and Mendelian randomisation analysis provide insights into the pathogenesis of heart failure. Nat Commun 11:163

29. Dupuis J, Langenberg C, Prokopenko I et al (2010) New genetic loci implicated in fasting glucose homeostasis and their impact on type 2 diabetes risk. Nat Genet 42:105-116

30. Willer CJ, Schmidt EM, Sengupta S et al (2013) Discovery and refinement of loci associated with lipid levels. Nat Genet 45:1274-1283

31. Locke AE, Kahali B, Berndt SI et al (2015) Genetic studies of body mass index yield new insights for obesity biology. Nature 518:197-206
32. Shungin D, Winkler TW, Croteau-Chonka DC et al (2015) New genetic loci link adipose and insulin biology to body fat distribution. Nature 518:187-196

33. Wood AR, Esko T, Yang J et al (2014) Defining the role of common variation in the genomic and biological architecture of adult human height. Nat Genet 46:1173-1186

34. Scott RA, Lagou V, Welch RP et al (2012) Large-scale association analyses identify new loci influencing glycemic traits and provide insight into the underlying biological pathways. Nat Genet 44:9911005

35. Burgess S, Bowden J, Fall T, Ingelsson E, Thompson SG (2017) Sensitivity analyses for robust causal inference from Mendelian randomization analyses with multiple genetic variants. Epidemiology 28:30-42

36. Verbanck M, Chen CY, Neale B, Do R (2018) Detection of widespread horizontal pleiotropy in causal relationships inferred from Mendelian randomization between complex traits and diseases. Nat Genet 50:693-698

37. Burgess S, Thompson SG (2015) Multivariable Mendelian randomization: the use of pleiotropic genetic variants to estimate causal effects. Am J Epidemiol 181:251-260

38. Spiller W, Davies NM, Palmer TM (2019) Software application profile: mrrobust-a tool for performing two-sample summary Mendelian randomization analyses. Int J Epidemiol 48:684-690

39. Yavorska OO, Burgess S (2017) MendelianRandomization: an R package for performing Mendelian randomization analyses using summarized data. Int J Epidemiol 46:1734-1739

40. Hemani G, Zheng J, Elsworth B et al (2018) The MR-base platform supports systematic causal inference across the human phenome. Elife 7:pii: e34408

41. Rajpathak SN, He M, Sun Q et al (2012) Insulin-like growth factor axis and risk of type 2 diabetes in women. Diabetes 61:2248-2254

42. Friedrich N, Thuesen B, Jorgensen $T$ et al (2012) The association between IGF-I and insulin resistance: a general population study in Danish adults. Diabetes Care 35:768-773

43. Larsson SC, Wolk K, Brismar K, Wolk A (2005) Association of diet with serum insulin-like growth factor I in middle-aged and elderly men. Am J Clin Nutr 81:1163-1167

44. Qin LQ, He K, Xu JY (2009) Milk consumption and circulating insulin-like growth factor-I level: a systematic literature review. Int J Food Sci Nutr 60(Suppl 7):330-340

45. Kazemi A, Speakman JR, Soltani S, Djafarian K (2019) Effect of calorie restriction or protein intake on circulating levels of insulin like growth factor I in humans: a systematic review and meta-analysis. Clin Nutr S0261-5614(0219):30312-30317

46. Bo Y, Liu C, Ji Z et al (2019) A high whey protein, vitamin D and E supplement preserves muscle mass, strength, and quality of life in sarcopenic older adults: a double-blind randomized controlled trial. Clin Nutr 38:159-164

47. Hill TR, Verlaan S, Biesheuvel E et al (2019) A vitamin D, calcium and leucine-enriched whey protein nutritional supplement improves measures of bone health in sarcopenic non-malnourished older adults: the PROVIDE study. Calcif Tissue Int 105:383-391

48. Fan M, Li Y, Wang C et al (2019) Dietary protein consumption and the risk of type 2 diabetes: a dose-response meta-analysis of prospective studies. Nutrients 11:pii: E2783

49. Lotta LA, Scott RA, Sharp SJ et al (2016) Genetic predisposition to an impaired metabolism of the branched-chain amino acids and risk of type 2 diabetes: a Mendelian randomisation analysis. PLoS Med 13: e1002179

Publisher's note Springer Nature remains neutral with regard to jurisdictional claims in published maps and institutional affiliations. 